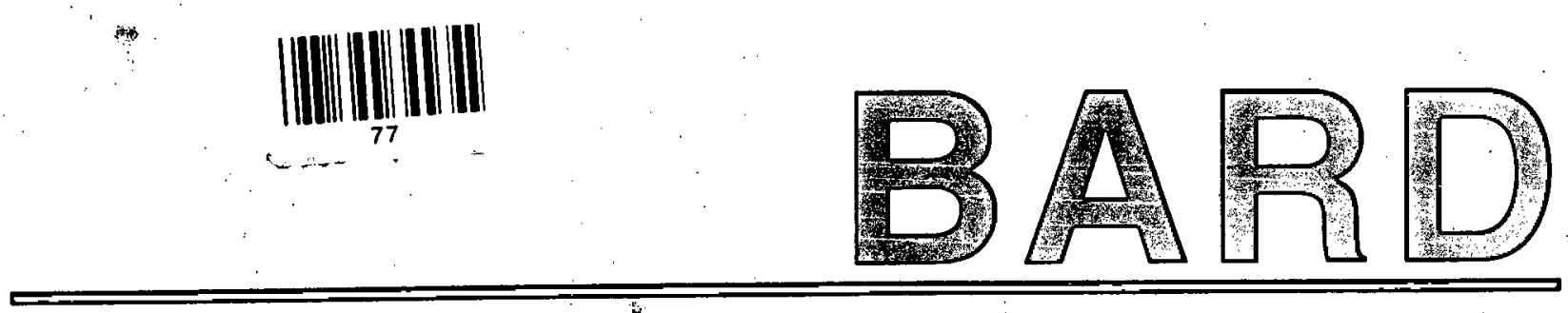

FINAL REPORT PROJECT NO. IS-1936-91

Host Adaptation in Entomopathogenic Nematodes: An Approach to Enhancing Biological Control Potential

I. Glazer, R. Gaugler, Y. Spiegel, E.E. Lewis 
Date: April 14, 1996

BARD Project Number: IS-1936-91

Project Title:

HOST ADAPTATION IN ENTOMOPATHOGENIC NEMATODES: AN APPROACH TO ENHANCING BIOLOGICAL CONTROL POTENTIAL

Investigators:

(Principal first)

\section{Glazer (PI)}

R. Gaugler

Y. Spiegel

E.E. LEWIS
Affiliated Institution:

A.R.O. Volcani Center

Rutgers University

A.R.O. Volcani Center

Rutgers University

Project Start Date: 20 Jul. 1992

Type of Report: 1st Annual

2nd Annual

Final $\mathbf{X}$

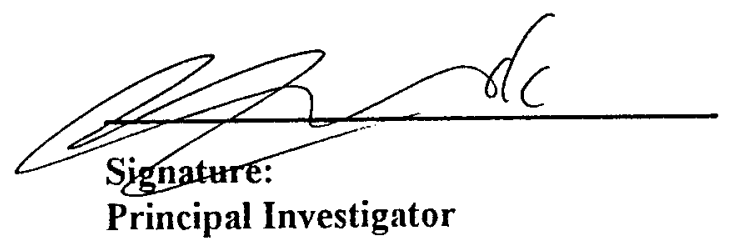

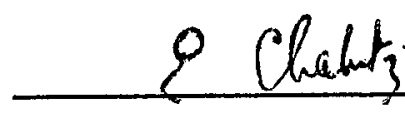

Signature:

Authorizing Official, Affiliated Institution

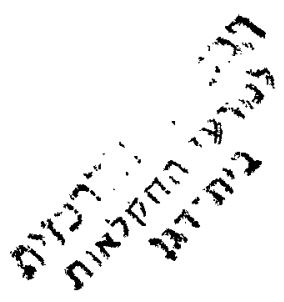




\section{TABLE OF CONTENTS}

Abstract............................

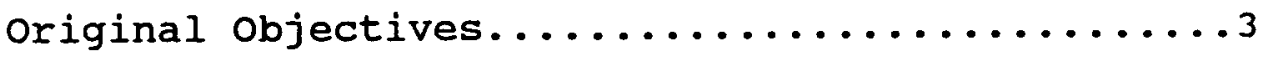

Relevant Data, Results and Discussion.........4

objective $1 \ldots \ldots \ldots \ldots \ldots \ldots \ldots \ldots \ldots \ldots$

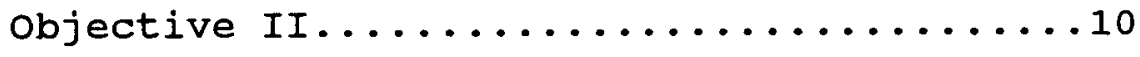

objective III.....................11

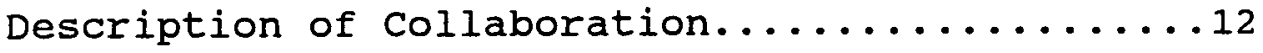

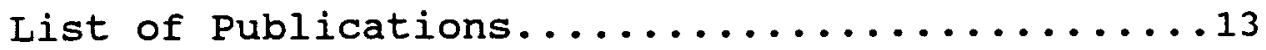




\section{ABSTRACT}

The overall objective of our research was to develop methods to match species of entomopathogenic nematodes against the insect pests which they would be best adapted to control. The underlying hypothesis for this work was that entomopathogenic nematodes should be most effective when used against insect species to which they are naturally adapted to parasitize. Toward this end, we undertook a number of related studies focusing primarily on nematode foraging strategies. We found that foraging strategies affected host associations directly and indirectly. Nematodes' responses to host cues, and the role of their sensory organs based on lectin binding, led to new approaches to determining host range for these parasites. Based on this work, we developed a laboratory bioassay of host recognition behavior designed to predict field results. We also determined that. nematodes that forage in a stationary manner (ambushers) have a slower metabolic rate than do active foragers (cruisers), thus their infective stage juveniles are longer lived. This study helps predict the duration of field activity after application and may partially explain field distributions of natural populations of entomopathogenic nematodes. The common thread linking all of these studies was that they led to a deeper understanding of the associations between entomopathogenic nematodes and insects as hosts. 


\section{ORIGINAL OBJECTIVES}

Our overall objective was to develop methods by which species and strains of entomopathogenic nematodes could be matched with target hosts for maximum control potential.

our Specific Aims were to:

1. Analyze host search and attachment behaviors.

2. Assess how energetics regulates host search.

3. Determine the biochemical and morphological bases of host recognition. 


\section{RELEVANT DATA, METHODS, RESULTS AND DISCUSSION}

\section{OBJECTIVE I.}

Foraging Behavior our first series of experiments was designed to assay the behavioral responses of nematodes with different strategies to potential hosts. We measured nematode responses to contact with cuticle or feces of larvae of the fall armyworm, spodoptera exigua, and the Japanese beetle, Popillia japonica. contact with the surface of green leaves served as a control. These tests compared $S$. carpocapsae (an ambush forager) with $S$. glaseri (a cruise forager). As anticipated, ambushing foragers did not respond as strongly to host contact as did cruise foragers. Further, $s$. glaseri responded most strongly to materials associated with $P$. japonica larvae, a reported natural host. We compared these same two nematode species for their response to volatile host cues and obtained similar results (Lewis et al. 1993). Carbon dioxide serves as an attractant for $S$. glaseri, whereas $S$. carpocapsae showed a minimal response to this material.

The behaviors we examined were very consistent, which led to a study showing that we could predict the foraging strategy of a nematode species by testing their behavioral response to host cues (Grewal et al. 1994). We hypothesized that when presented with cues from a remote host, mobile foragers should respond positively and ambushing foragers would not respond. This hypothesis was tested with steinernema anomali, s. carpocapsae, 
Steinernema feltiae, S. glaseri, steinernema riobravis, Steinernema scapterisci, Heterorhabditis bacteriophora and Heterorhabditis megidis. Indeed, when these tests were performed on very mobile cruising foragers ( $S$. glaseri or $H$. bacteriophora, for example), the response to remote hosts was very strong. Conversely, when testing ambushing foragers ( $S$. carpocapsae or $S$. scapterisci) their response was nil. However, when testing nematodes with foraging strategies that were intermediate between these two extremes ( $S$. feltiae or $s$. riobravis), the results were not as clearly predictive.

The importance of the order of host cues to nematodes with different foraging strategies was also compared. We found that S. carpocapsae, an ambush forager, aggregated at the source of volatile host cues only after attachment to host cuticle. They also parasitized hosts more quickly after contact with cuticle. These results suggested that host recognition occurred for $s$. carpocapsae while the nematode was on the cuticle of the host. steinernema glaseri was unaffected by exposure to combinations of host cues; they responded similarly when cues are offered singly or in series. Steinernema feltiae, a nematode with characteristics of both ambushing and cruising, was affected by cue hierarchies when either contact or volatile cues were presented first. We concluded that an ambusher's response to host materials has a more fixed contextual framework than a cruiser's due to a more predictable order of events (Lewis et al. 1995). 
Three assays of host recognition behavior have been designed, based upon the foraging behavior studies described above. The utility of such assays is based on two hypotheses: 1) Entomopathogenic nematodes should be effective controlling insects to which they are naturally adapted, and 2) Recognition behavior (once determined) should be most pronounced when exposed to materials from natural hosts. Our study tested the second of these hypotheses directly. The assays were based upon the nematodes' respective responses to host cues. First, we tested whether host recognition by entomopathogenic nematodes occurs through contact with insect gut contents and whether this recognition is linked to successful infection. The responses of four entomopathogenic nematode species: $H$. bacteriophora, $S$. carpocapsae, S. glaseri and $S$. scapterisci were studied during contact with the gut contents of 4 species of insects: Acheta domesticus, Blatella germanica, P. japonica and $S$. exigua. Not surprisingly, we found that only cruising nematodes demonstrated any response to the host materials tested. There was a relationship between one recognition behavior, head thrusting, by S. glaseri and $H$. bacteriophora, to infectivity towards the various host species tested (Grewal et al. 1993). However, the practicality of this assay is questionable since "head thrusting" is a complex behavior, and may be plagued by subjective interpretation when this test is performed in other laboratories. A related assay for $s$. glaseri is currently under development, based upon this nematode's response to host cuticle, 
described above. Preliminary evidence shows that giving-up-time, an index of behavioral changes in nematode searching behavior due to information about a host, is related to host suitability. We are again testing a variety of different perspective hosts and will attempt to corellate host suitability with the measured behavior.

A third assay, based upon the hierarchical response of $S$. carpocapsae to host cues has proven an accurate predictor of host suitability. Infective juveniles respond to volatile cues after contact with host cuticle. The response stimulated by the cuticle of different insect species correlates significantly with the level of reproduction supported by the test hosts, and with nematode-induced host mortality for that insect species (manuscript in review). The black cutworm, Agrotis ipsilon, stimulated the greatest behavioral response and also supported the highest reproductive levels of the insects tested. Conversely, non-insect arthropods (Diplopoda and Isopoda) stimulated no recognition response by $S$. carpocapsae and do not serve as hosts.

Sex Ratio We have determined that the sexes of steinernematids behave differently from one another with respect to their response to host cues, their assessment of host quality and their emergence from the host cadaver. Males are more sensitive to volatile host cues than are females, and tend to find the host first. Further, we demonstrated a relationship between nematode 
foraging strategy and their pattern of emergence from the host. Cruising species, such as S. glaseri, are protandrous (males emerge from the cadaver before females) whereas ambushing species like $S$. carpocapsae are not. In $S$. glaseri, we also found that newly-emerged females were attracted strongly to hosts that were pre-parasitized by males, whereas healthy hosts elicited only weak attraction. After a week, the females were strongly attracted to pre-parasitized and healthy hosts. These results may explain some of the reported variability among different harvests of $S$. glaseri seen in laboratory experiments and field trials (Lewis and Gaugler 1994).

To determine whether there is a genetic component to sex ratio, we conducted a selection study with $s$. glaseri. We selected the early component of the nematode harvest which were mostly males (nematodes emerging the first day of the harvest) , and the late component, which were primarily females (those nematodes emerging after day 7). After 12 rounds of selection (ca. 25 generations), the pattern of emergence of early and late, lines were significantly different from each other and from the base strain, suggesting a genetic component to nematode emergence from the cadaver (manuscript in review).

colonization stimulated by our studies of protandry, described above, we conducted several experiments to assess the effect of previous parasitization (both by conspecific and heterospecific nematodes) on the attractiveness of hosts to infective juveniles. 
The effects of previous infection were different for various entomopathogenic nematode species. Steinernema carpocapsae infective juveniles showed increased attraction to hosts with conspecific infections, as did $S$. glaseri and $S$. feltiae. However, $S$. carpocapsae were repelled by hosts pre-infected with s. glaseri. Steinernema glaseri, on the other hand, responded to hosts infected with $S$. carpocapsae as strongly as they did to non-infected hosts. We and others have suggested that nematode species that are strong competitors inside the host may not be repelled by heterospecific infections. This work may shed light on the resource partitioning scenarios that must exist in entomopathogenic nematode communities in the field in either natural mixed populations or in areas where applied nematodes are added to extant native populations (Grewal et al. manuscript in review).

Mating and Reproductive Isolation We described the mating behaviors of $S$. carpocapsae and $S$. feltiae. For the first time, evidence of a mating pheromone for entomopathogenic nematodes was presented. The pheromone is produced by the female adult while inside the host and is attractive to adult males. The pheromone is species-specific (male $S$. carpocapsae are not attracted to female $S$. feltiae and vice versa). Also, males are only attracted to virgin conspecific females, suggesting that females mate only once (Lewis et al. manuscript in preparation). Decisions occurring during development and mating inside the host 
can influence host finding and host assessment profoundly.

\section{OBJECTIVE II.}

Aging Infective juvenile behavior changes with age. We conducted a study asking two questions: 1) what is the relationship between metabolic rate, energy reserves and foraging strategy and 2) When a foraging strategy fails, will an infective juvenile switch strategies (will an ambusher become a cruizer)? Infective juveniles of $S$. carpocapsae, $S$. glaseri, and $H$. bacteriophora were stored in water and changes in their behavior, metabolic rate, energy reserves, and infectivity were measured throughout the storage period. Steinernema carpocapsae was least active and maintained the lowest metabolic rate, due to its ambushing foraging strategy and lived as long as 12 weeks in storage. Heterorhabditis bacteriophora was more active and had the highest metabolic rate and lived only a maximum of 7 weeks in storage. Steinernema glaseri was most active and had an intermediate metabolic rate and lived nearly 20 weeks in storage. Neither cruising species changed foraging strategy for the duration of the study. Steinernema carpocapsae decreased nictation rate and increased their locomotory rate with age. Any change in searching strategy occurred without assessment of the profitability or distribution of potential hosts. Upon further investigation, the increase in locomotion by $s$. carpocapsae did not render it a more effective mobile forager. 


\section{OBJECTIVE III.}

Lectins were used to determine the location of carbohydrate exudates on the surface of three species of entomopathogenic nematodes (S. carpocapsae, H. bacteriophora and $S$. glaseri) with different foraging strategies. The degree of labelling to the areas near sensory organs was related to the importance of carbohydrates in host finding and recognition. Several lectins were screened with various degrees of binding to nematodes. Seven different lectins bound to carbohydrates that covered the cuticle, but not specifically the amphids of $s$. carpocapsae. only wheat germ agglutinin bound specifically to both the anterior region of $H$. bacteriophora and to the anterior and posterior of $S$. glaseri. Wheat germ agglutinin treatment increased the rate of attachment during ambushing by $s$. carpocapsae, and this effect was negated by treatment with Nacetylglucosamine, a competing sugar. The infectivity of $S$. glaseri in an assay that required movement through $5 \mathrm{~cm}$ of sand to find the host was decreased by wheat germ agglutinin treatment. The work started here with lectins is continuing in both laboratories. 


\section{DESCRIPTION OF THE COLLABORATION}

We have been fortunate to have a long-term relationship between the American laboratory, directed by Dr. Randy Gaugler and the Israeli laboratory, directed by Dr. Itamar Glazer. During the early stages of the research, all personnel maintained close contact via e-mail and the telephone. We frequently shared techniques, data, slides, problems, break-throughs and news throughout the duration of the project. In addition, Dr. Glazer visited the American laboratory for 12 months during the project. This facilitated the true collaborative nature of the research, and stimulated planning for future collaborative efforts among all of the principal investigators. We shared laboratory space and expertise during this visit in a way that was impossible through the telephone or electronic mail. This collaborative research added strength to the already significant ties between these two laboratories. Future collaboration is certain. 


\section{LIST OF PUBLICATIONS ASSOCIATED FITH THIS BARD PROJECT}

\section{Published}

Lewis, E.E., I. Glazer \& R Gaugler. 1996. The location and behavioral effects of lectin binding on entomopathogenic nematodes with different foraging strategies. Journal of Chemical Ecology. In press.

Lewis, E.E., S. Selvan, J.F. Campbell \& R. Gaugler. 1995. Changes in foraging behaviour during the infective stage of entomopathogenic nematodes. Parasitology. 110: 583-590.

Lewis, E.E., P.S. Grewal \& R. Gaugler. 1995. Hierarchical order of host cues in parasite foraging strategies. Parasitology. 110: $207-213$

Lewis, E.E. \& R. Gaugler. 1994. Entomopathogenic nematode sexratio relates to foraging strategy. Journal of Invertebrate Pathology. 64: 238-242.

Lewis, E.E. 1994. Foraging strategies as a theoretical framework for the study of entomopathogenic nematode life history traits. In: Proceedings of the 6th International colloquium on Invertebrate Pathology and Microbial Control. 109-114. 
Grewal, P.S., E.E. Lewis, J.F. Campbell \& R. Gaugler. 1994 searching behavior as a predictor of foraging strategy for entomopathogenic nematodes. Parasitology. 108: 207-215.

Grewal, P.S., R. Gaugler \& E.E. Lewis. 1993. Host recognition behavior by entomopathogenic nematodes during contact with gut contents and its adaptive significance. Journal of Parasitology. 79: 495-503.

Lewis, E.E., R. Gaugler \& R. Harrison. 1993. Response of cruiser and ambusher entomopathogenic nematodes (Steinernematidae) to host volatile cues. Canadian Journal of Zoology. 71: 765-769.

\section{submitted Manuscripts}

Lewis, E.E., Ricci, M. \& R. Gaugler. Host recognition behaviour relates to host suitability for steinernema carpocapsae (Rhabditida: steinernematidae). Submitted to Parasitology.

Lewis, E.E., Campbell, J.F. \& R. Gaugler. The effects of aging on steinernema carpocapsae foraging behavior. Submitted to Nematologica.

Grewal, P.S., E.E. Lewis \& R. Gaugler. Evidence for reproductive isolation in non-reproductive infective stages of entomopathogenic nematodes (Rhabditida: steinernematidae). Submitted to Journal of Chemical Ecology. 
Stuart, R., E.E. Lewis \& R. Gaugler. Genetic selection alters emergence characteristics in the entomopathogenic nematode, steinernema glaseri. Submitted to Parasitology.

\section{Manuscripts in Preparation}

Lewis, E.E., Barbarosa, B. \& R. Gaugler. Pheromone-mediated mating behavior of Steinernematidae. For submission to Journal of Chemical Ecology. 